\title{
A flipped 331 model
}

\author{
Renato M. Fonseca and Martin Hirsch \\ AHEP Group, Instituto de Física Corpuscular, C.S.I.C./Universitat de València, \\ Parc Científic de Paterna. Calle Catedrático José Beltrán, \\ 2 E-46980 Paterna (Valencia), Spain \\ E-mail: renato.fonseca@ific.uv.es, mahirsch@ific.uv.es
}

ABstract: Models based on the extended $\mathrm{SU}(3)_{C} \times \mathrm{SU}(3)_{L} \times \mathrm{U}(1)_{X}$ (331) gauge group usually follow a common pattern: two families of left-handed quarks are placed in antitriplet representations of the $\mathrm{SU}(3)_{L}$ group; the remaining quark family, as well as the left-handed leptons, are assigned to triplets (or vice-versa). In this work we present a flipped 331 model where this scheme is reversed: all three quark families are in the same representation and it is the lepton families which are discriminated by the gauge symmetry. We discuss fermion masses and mixing, as well as $Z^{\prime}$ interactions, in a minimal model implementing this idea.

Keywords: Beyond Standard Model, Gauge Symmetry, Neutrino Physics

ArXiv EPRINT: 1606.01109 


\section{Contents}

1 Introduction 1

2 A flipped 331 model 3

3 Fermion masses and FCNC constraints 5

$\begin{array}{lll}4 & \text { Summary } & 10\end{array}$

\section{Introduction}

The idea of embedding the Standard Model (SM) electroweak symmetry group in $\mathrm{SU}(3)_{L} \times$ $\mathrm{U}(1)_{X}$ was proposed a long time ago. Early work, see for example [1-4], were mostly attempts to explain the absence of flavor changing neutral currents (FCNCs) using an extended gauge sector instead of a GIM mechanism. A fully-fledged three generation model was then proposed by Singer, Valle and Schechter (SVS) in 1980 [5]. A "minimal" 331 model was constructed by Pisano, Pleitez [6] and Frampton [7] (PPF). Both the SVS and the PPF models, as well as (nearly) all other 331 models, follow the same, simple construction principles, as discussed below.

Any model beyond the SM needs to satisfy two important constraints:

1. Extra fermions should not be introduced, unless they are vector-like under the SM group; i.e. they must either be in a real representation of $\mathrm{SU}(3)_{C} \times \mathrm{SU}(2)_{L} \times \mathrm{U}(1)_{Y}$, or come in pairs of complex conjugated representations.

2. Gauge anomalies should cancel.

These two conditions turn out to be very restrictive for the fermion sector of 331 models. Just to mention one example, the authors of [8] considered the consequences of gauge anomaly cancellation (condition 2) in 331 models with $\mathrm{SU}(3)_{L}$ singlets, triplets and antitriplets. With only those representations the standard construction is to place the lefthanded lepton doublets of the $\mathrm{SM}$ in three $\mathrm{SU}(3)_{L}$ triplets $\psi_{\ell}$ (one per generation). Then, in order to cancel the $\mathrm{SU}(3)_{L}^{3}$ anomaly, for the left-handed quarks there is no choice except to put two families in anti-triplets $\left(Q_{12}\right)$ and the remaining one $\left(Q_{3}\right)$ in an $\mathrm{SU}(3)_{L}$ triplet (due to the color multiplicity of the quarks). Furthermore, one should add $\mathrm{SU}(3)_{L}$ singlets for the right-landed leptons and quarks $\left(\ell^{c}, u^{c}\right.$ and $\left.d^{c}\right)$. The fermion content of the model is wrapped up by noting that each $\mathrm{SU}(3)_{L}$ (anti)triplet contains an $\mathrm{SU}(2)_{L}$ doublet plus an extra singlet, and this latter fermion must be vector-like, hence $\mathrm{SU}(3)_{L}$ singlets with an adequate color and $\mathrm{U}(1)_{X}$ charge should be added to the model $\left(\ell_{X}, J_{12}\right.$ and $\left.J_{3}\right)$. 


\begin{tabular}{|cccc|}
\hline Name & 331 representation & SM group decomposition & \# flavors \\
\hline$\psi_{\ell}$ & $\left(\mathbf{1}, \mathbf{3},-\frac{1}{2}-\frac{1}{2 \sqrt{3}} \beta\right)$ & $\left(\mathbf{1}, \widehat{\mathbf{2}},-\frac{1}{2}\right)+\left(\mathbf{1}, \widehat{\mathbf{1}},-\frac{1}{2}-\frac{\sqrt{3}}{2} \beta\right)$ & 3 \\
$\ell^{c}$ & $(\mathbf{1}, \mathbf{1}, 1)$ & $(\mathbf{1}, \widehat{\mathbf{1}}, 1)$ & 3 \\
$\ell_{X}$ & $\left(\mathbf{1}, \mathbf{1}, \frac{1}{2}+\frac{\sqrt{3}}{2} \beta\right)$ & $\left(\mathbf{1}, \widehat{\mathbf{1}}, \frac{1}{2}+\frac{\sqrt{3}}{2} \beta\right)$ & 3 \\
$Q_{12}$ & $\left(\mathbf{3}, \overline{\mathbf{3}}, \frac{1}{6}+\frac{1}{2 \sqrt{3}} \beta\right)$ & $\left(\mathbf{3}, \widehat{\mathbf{2}}, \frac{1}{6}\right)+\left(\mathbf{3}, \widehat{\mathbf{1}}, \frac{1}{6}+\frac{\sqrt{3}}{2} \beta\right)$ & 2 \\
$Q_{3}$ & $\left(\mathbf{3}, \mathbf{3}, \frac{1}{6}-\frac{1}{2 \sqrt{3}} \beta\right)$ & $\left(\mathbf{3}, \widehat{\mathbf{2}}, \frac{1}{6}\right)+\left(\mathbf{3}, \widehat{\mathbf{1}}, \frac{1}{6}-\frac{\sqrt{3}}{2} \beta\right)$ & 1 \\
$u^{c}$ & $\left(\overline{\mathbf{3}}, \mathbf{1},-\frac{2}{3}\right)$ & $\left(\overline{\mathbf{3}}, \widehat{\mathbf{1}},-\frac{2}{3}\right)$ & 3 \\
$d^{c}$ & $\left(\overline{\mathbf{3}}, \mathbf{1}, \frac{1}{3}\right)$ & $\left(\overline{\mathbf{3}}, \widehat{\mathbf{1}}, \frac{1}{3}\right)$ & 3 \\
$J_{12}$ & $\left(\overline{\mathbf{3}}, \mathbf{1},-\frac{1}{6}-\frac{\sqrt{3}}{2} \beta\right)$ & $\left(\overline{\mathbf{3}}, \widehat{\mathbf{1}},-\frac{1}{6}-\frac{\sqrt{3}}{2} \beta\right)$ & 2 \\
$J_{3}$ & $\left(\overline{\mathbf{3}}, \mathbf{1},-\frac{1}{6}+\frac{\sqrt{3}}{2} \beta\right)$ & $\left(\overline{\mathbf{3}}, \widehat{\mathbf{1}},-\frac{1}{6}+\frac{\sqrt{3}}{2} \beta\right)$ & 1 \\
\hline$\phi_{1}$ & $\left(\mathbf{1}, \mathbf{3}, \frac{1}{2}-\frac{1}{2 \sqrt{3}} \beta\right)$ & $\left(\mathbf{1}, \widehat{\mathbf{2}}, \frac{1}{2}\right)+\left(\mathbf{1}, \widehat{\mathbf{1}}, \frac{1}{2}-\frac{\sqrt{3}}{2} \beta\right)$ & 1 \\
$\phi_{2}$ & $\left(\mathbf{1}, \mathbf{3}, \frac{1}{\sqrt{3}} \beta\right)$ & $\left(\mathbf{1}, \widehat{\mathbf{2}}, \frac{\sqrt{3}}{2} \beta\right)+(\mathbf{1}, \widehat{\mathbf{1}}, 0)$ & 1 \\
$\phi_{3}$ & $\left(\mathbf{1}, \mathbf{3},-\frac{1}{2}-\frac{1}{2 \sqrt{3}} \beta\right)$ & $\left(\mathbf{1}, \widehat{\mathbf{2}},-\frac{1}{2}\right)+\left(\mathbf{1}, \widehat{\mathbf{1}},-\frac{1}{2}-\frac{\sqrt{3}}{2} \beta\right)$ & 1 \\
\hline
\end{tabular}

Table 1. Representations used in a "standard" 331 model for a generic $\beta$ parameter. The $\phi_{i}$ are scalars while all other fields are left-handed Weyl fermions. It is also possible to flip the sign of $\beta$ and at the same time swap all $\mathrm{SU}(3)_{L}$ representations to the corresponding anti-representations.

Finally, for the symmetry breaking and the generation of fermion masses, a set of scalars is needed. Again, in the minimal setups, usually three $\mathrm{SU}(3)_{L}$ triplets are introduced to provide the required Yukawa interactions and gauge boson masses.

It is important to note that $\mathrm{SU}(2)_{L} \times \mathrm{U}(1)_{Y}$ can be embedded in $\mathrm{SU}(3)_{L} \times \mathrm{U}(1)_{X}$ in more than one way; this can be parametrized by a number $\beta$ which relates the hypercharge $Y$, the $T_{8}$ generator of $\mathrm{SU}(3)_{L}$ and $X$ as follows:

$$
Y=\beta T_{8}+X
$$

In fact, it turns out that one can build minimal models obeying the two conditions above for an arbitrary $\beta$ with the representations in table $1 .^{1}$

For example, the Singer-Valle-Schechter model [5] is obtained by substituting $\beta=$ $-1 / \sqrt{3}$, while the Pisano-Pleitez-Frampton model $[6,7]$ uses $\beta=-\sqrt{3}$. The various 331 models may also differ from one-another in the scalar sector or perhaps due to the addition of vector fermions (under the full 331 group) — indeed, such modifications might be needed to account for the observed charged lepton [10] and neutrino masses [11]. Furthermore, for some values of $\beta$, some of the representations in the table might be vector-like (or form vector-like pairs) hence they might be removed without violating the conditions 1 and 2

\footnotetext{
${ }^{1}$ Throughout this work $\mathrm{SU}(2)_{L}$ representations have hats to distinguish them from $\mathrm{SU}(3)_{L}$ representations.
} 
in the text. This reduces the number of fermion fields in the SVS model to 18 and in the PPF version to 15 .

Switching triplets with anti-triplets of $\mathrm{SU}(3)_{L}$ and changing $\beta$ to $-\beta$ is also allowed and this leads to pairs of models, with rather similar phenomenology but subtly different FCNC effects, suppressed by $\left(\Lambda_{E W} / \Lambda_{331}\right)^{2}$ [9]. Ignoring running effects, it is easy to show that the gauge couplings of $\mathrm{SU}(3)_{L} / \mathrm{SU}(2)_{L}, \mathrm{U}(1)_{Y}$ and $\mathrm{U}(1)_{X}$ obey the relation $g_{Y}^{-2}=g_{X}^{-2}+\beta^{2} g_{L}^{-2}$ therefore the requirement that $g_{X}^{2}$ is positive implies that $|\beta| \leq g_{L} / g_{Y}=\tan ^{-1} \theta_{W} \approx 1.8$. If we add the condition that no fractionally charged leptons should appear in the physical spectrum, ${ }^{2}$ only four values of $\beta$ lead to viable models: $\beta= \pm \sqrt{3}, \pm 1 / \sqrt{3}$. With the abovementioned switch to anti-representations this makes a total of 8 possible basic models.

Finally we mention the 331 model which is obtainable from an $E_{6}$ grand unified theory [12]. This model is a notable exception to the scheme of table 1 because in it all three families of quarks and leptons are in equal $\mathrm{SU}(3)_{C} \times \mathrm{SU}(3)_{L} \times \mathrm{U}(1)_{X}$ representations. It is curious to note that in this model anomaly cancellation occurs within one family - as in the Standard Model - thus dispelling the notion that 331 models predict the number of families to be equal to the number of colours.

\section{$2 \quad$ A flipped 331 model}

As we have mentioned already, in addition to choosing different values of $\beta$, some simple adjustments can be made to the class of 331 models mentioned so far: (a) the scalar sector may be changed, for example by adding more fields or (b) fermions which are vector-like under the full 331 symmetry might be added to the model. More interesting, however, is the observation that gauge anomaly cancellation does not force 331 models to follow the structure of table 1 . Indeed, the field content in table 2 is not only anomaly free; it has in fact the same total number of fermion representations as the standard 331 model with the same $\beta$. Since in this model there is perfect quark family replication, while leptons are placed into different representations, we call this setup a flipped 331 model.

Anomaly cancellation works in the flipped 331 model as follows: an $\mathrm{SU}(3)_{L}$ sextet contributes as much as 7 triplets to the $\mathrm{SU}(3)_{L}^{3}$ anomaly, which at first seems problematic. Not only is this number large, it is not directly relatable to the number of families (3) nor the number of colors (also 3 ). The simplest solution to such a large anomaly contribution is to make the sextet field a lepton; otherwise, if it were a quark, the color multiplicity would compound the problem of canceling the $\mathrm{SU}(3)_{L}^{3}$ anomaly. The decomposition of a sextet representation is as follows (for a generic $\beta$ ):

$$
(\mathbf{1}, \mathbf{6}, x) \rightarrow\left(\mathbf{1}, \widehat{\mathbf{3}}, x+\frac{1}{\sqrt{3}} \beta\right)+\left(\mathbf{1}, \widehat{\mathbf{2}}, x-\frac{1}{2 \sqrt{3}} \beta\right)+\left(\mathbf{1}, \widehat{\mathbf{1}}, x-\frac{2}{\sqrt{3}} \beta\right) .
$$

Significantly, the sextet contains an $\mathrm{SU}(2)_{L}$ triplet. This triplet will have necessarily an electroweak scale mass, unless it is made a vector particle under the SM gauge group. To do that one could introduce other (bigger) $\mathrm{SU}(3)_{L}$ multiplets to find a $\left(\mathbf{1}, \widehat{\mathbf{3}},-x-\frac{1}{\sqrt{3}} \beta\right)$

\footnotetext{
${ }^{2}$ Fractionally charged leptons, produced abundantly in the early universe, would lead to an unacceptable cosmology, because there are no decay modes for the lightest of these exotic states.
} 


\begin{tabular}{|c|c|c|c|c|}
\hline Name & 331 rep. & SM group decomposition & Components & \# flavors \\
\hline$L_{e}$ & $\left(\mathbf{1}, \mathbf{6},-\frac{1}{3}\right)$ & $(\mathbf{1}, \widehat{\mathbf{3}}, 0)+\left(\mathbf{1}, \widehat{\mathbf{2}},-\frac{1}{2}\right)+(\mathbf{1}, \widehat{\mathbf{1}},-1)$ & $\left(\begin{array}{ccc}\Sigma^{+} & \frac{1}{\sqrt{2}} \Sigma^{0} & \frac{1}{\sqrt{2}} \nu_{e} \\
\frac{1}{\sqrt{2}} \Sigma^{0} & \Sigma^{-} & \frac{1}{\sqrt{2}} \ell_{e} \\
\frac{1}{\sqrt{2}} \nu_{e} & \frac{1}{\sqrt{2}} \ell_{e} & E_{e}\end{array}\right)$ & 1 \\
\hline$L_{\alpha=\mu, \tau}$ & $\left(\mathbf{1}, \mathbf{3},-\frac{2}{3}\right)$ & $\left(\mathbf{1}, \widehat{\mathbf{2}},-\frac{1}{2}\right)+(\mathbf{1}, \widehat{\mathbf{1}},-1)$ & $\left(\nu_{\alpha}, \ell_{\alpha}, E_{\alpha}\right)^{T}$ & 2 \\
\hline$\ell_{\alpha}^{c}$ & $(\mathbf{1}, \mathbf{1}, 1)$ & $(\mathbf{1}, \widehat{1}, 1)$ & $\ell_{\alpha}^{c}$ & 6 \\
\hline$Q_{\alpha}$ & $\left(\mathbf{3}, \overline{\mathbf{3}}, \frac{1}{3}\right)$ & $\left(\mathbf{3}, \widehat{\mathbf{2}}, \frac{1}{6}\right)+\left(\mathbf{3}, \widehat{\mathbf{1}}, \frac{2}{3}\right)$ & $\left(d_{\alpha},-u_{\alpha}, U_{\alpha}\right)^{T}$ & 3 \\
\hline$u_{\alpha}^{c}$ & $\left(\overline{\mathbf{3}}, \mathbf{1},-\frac{2}{3}\right)$ & $\left(\overline{\mathbf{3}}, \widehat{\mathbf{1}},-\frac{2}{3}\right)$ & $u_{\alpha}^{c}$ & 6 \\
\hline$d_{\alpha}^{c}$ & $\left(\overline{\mathbf{3}}, \mathbf{1}, \frac{1}{3}\right)$ & $\left(\overline{\mathbf{3}}, \widehat{\mathbf{1}}, \frac{1}{3}\right)$ & $d_{\alpha}^{c}$ & 3 \\
\hline$\phi_{i=1,2}$ & $\left(\mathbf{1}, \mathbf{3}, \frac{1}{3}\right)$ & $\left(\mathbf{1}, \widehat{\mathbf{2}}, \frac{1}{2}\right)+(\mathbf{1}, \widehat{\mathbf{1}}, 0)$ & $\left(H_{i}^{+}, H_{i}^{0}, \sigma_{i}^{0}\right)^{T}$ & 2 \\
\hline$\phi_{3}$ & $\left(\mathbf{1}, \mathbf{3},-\frac{2}{3}\right)$ & $\left(\mathbf{1}, \widehat{\mathbf{2}},-\frac{1}{2}\right)+(\mathbf{1}, \widehat{\mathbf{1}},-1)$ & $\left(H_{3}^{0}, H_{3}^{-}, \sigma_{3}^{-}\right)^{T}$ & 1 \\
\hline$S$ & $\left(\mathbf{1}, \mathbf{6}, \frac{2}{3}\right)$ & $(\mathbf{1}, \widehat{\mathbf{3}}, 1)+\left(\mathbf{1}, \widehat{\mathbf{2}}, \frac{1}{2}\right)+(\mathbf{1}, \widehat{\mathbf{1}}, 0)$ & $\left.\begin{array}{ccc}\Delta^{++} & \frac{1}{\sqrt{2}} \Delta^{+} & \frac{1}{\sqrt{2}} H_{S}^{+} \\
\frac{1}{\sqrt{2}} \Delta^{+} & \Delta^{0} & \frac{1}{\sqrt{2}} H_{S}^{0} \\
\frac{1}{\sqrt{2}} H_{S}^{+} & \frac{1}{\sqrt{2}} H_{S}^{0} & \sigma_{S}^{0}\end{array}\right)$ & 1 \\
\hline
\end{tabular}

Table 2. Representations for the flipped 331 model. The $\phi$ 's and $S$ are scalars while all other fields are left-handed Weyl fermions. The letters $i$ and $\alpha$ stand for flavor indices, going from 1 to the number quoted in the last column (except for $L_{\alpha=\mu, \tau}$ ). The components of the $\mathrm{SU}(3)_{L}$ triplets in the forth column match the ordering of the SM group decomposition given in the second column; as for the sextets, $\Sigma$ and $\Delta$ stand for the triplet sub-representations, $\left(\nu_{e}, \ell_{e}\right)^{T}$ and $\left(H_{S}^{+}, H_{S}^{0}\right)^{T}$ form $\mathrm{SU}(2)_{L}$ doublets, while $E_{e}$ and $\sigma_{S}^{0}$ are singlets.

state which can form a vector pair with the triplet in equation (2.1). While such a construction might indeed be possible, it runs the risk of requiring a large number of extra representations (see section 3.6 of [13]). Yet, there is a simpler alternative. For $x=-\frac{1}{\sqrt{3}} \beta$ the triplet in equation (2.1) becomes a real representation, thereby solving the problem. Note that, if the sextet was a quark, this idea would not work.

We will thus focus on the possibility of identifying the doublet inside the sextet as one of the families of the left-handed leptons: $\widehat{L}_{e}=(\mathbf{1}, \widehat{\mathbf{2}},-1 / 2)$. (The reason for associating it with the electron will be made clear in the next section.) In that case, $x-\frac{1}{2 \sqrt{3}} \beta=-\frac{\sqrt{3}}{2} \beta$ should equate to $-1 / 2$, hence

$$
\beta=\frac{1}{\sqrt{3}}
$$

The two remaining families of left-handed leptons, $\widehat{L}_{\mu}$ and $\widehat{L}_{\tau}$, can then be put into either triplets or anti-triplets of $\mathrm{SU}(3)_{L}$. However, the triplet possibility is clearly more interesting for the following reason: one sextet and two triplets provide an anomaly contribution exactly equal to the one of nine triplets. Hence, if all three left-handed quarks are placed in anti-triplets of $\mathrm{SU}(3)_{L}$, the $\mathrm{SU}(3)_{L}^{3}$ cancels. It is then rather simple to add the correct 


\begin{tabular}{|ccccccc|}
\hline Field & $\mathrm{SU}(3)_{C}^{3}$ & $\mathrm{SU}(3)_{L}^{3}$ & $\mathrm{SU}(3)_{C}^{2} \mathrm{U}(1)_{X}$ & $\mathrm{SU}(3)_{L}^{2} \mathrm{U}(1)_{X}$ & $\mathrm{U}(1)_{X}^{3}$ & \# flavors \\
\hline$L_{e}$ & 0 & $\frac{7}{2}$ & 0 & $-\frac{5}{6}$ & $-\frac{2}{9}$ & 1 \\
$L_{\alpha=\mu, \tau}$ & 0 & $\frac{1}{2}$ & 0 & $-\frac{1}{3}$ & $-\frac{8}{9}$ & 2 \\
$\ell_{\alpha}^{c}$ & 0 & 0 & 0 & 0 & 1 & 6 \\
$Q_{\alpha}$ & $\frac{3}{2}$ & $-\frac{3}{2}$ & $\frac{1}{2}$ & $\frac{1}{2}$ & $\frac{1}{3}$ & 3 \\
$u_{\alpha}^{c}$ & $-\frac{1}{2}$ & 0 & $-\frac{1}{3}$ & 0 & $-\frac{8}{9}$ & 6 \\
$d_{\alpha}^{c}$ & $-\frac{1}{2}$ & 0 & $\frac{1}{6}$ & 0 & $\frac{1}{9}$ & 3 \\
\hline
\end{tabular}

Table 3. Contribution of one copy/flavor of a given fermion representation to each of the five gauge anomalies. The sum of all rows, weighted by the number of flavors in the last column, adds up to zero, hence the model is anomaly free.

$\mathrm{SU}(3)_{L}$ singlets in order to reproduce the SM chirality (condition 1 above). And, surprisingly, without further tuning the model, it turns out that not only does the $\mathrm{SU}(3)_{L}^{3}$ gauge anomaly cancel, but in fact all gauge anomalies cancel in this minimal setup (see table 3). Thus, no additional particles are needed in the fermion sector of this minimal flipped model, which is summarized in table 2.

With conditions 1 and 2 satisfied, all that remains is to find adequate scalar fields. Using the notation in table 2, one should have at least the Yukawa interactions $Q u^{c} \times$ scalar, $Q d^{c} \times$ scalar, $L_{\mu \tau} \ell^{c} \times$ scalar, $L_{e} L_{e} \times$ scalar and $L_{e} \ell^{c} \times$ scalar. The minimal setup to do this contains three scalar triplets $\phi_{1,2,3}$ and a scalar sextet $S$. Note that two copies of the same triplet representation, $\phi_{1}$ and $\phi_{2}$, are needed for fermion masses, as explained below.

\section{Fermion masses and FCNC constraints}

We shall not consider in detail the scalar potential of the model. Instead we assume that there exists a stable, charge-preserving vacuum state and study the consequences. In this spirit, we allow all neutral scalar components to have a non-zero vacuum expectation value $(\mathrm{VEV})$ :

$$
\left\langle\sigma_{1,2}^{0}\right\rangle \equiv n_{1,2},\left\langle H_{1,2,3}^{0}\right\rangle \equiv k_{1,2,3},\left\langle\sigma_{S}^{0}\right\rangle \equiv n_{S},\left\langle H_{S}^{0}\right\rangle \equiv k_{S},\left\langle\Delta^{0}\right\rangle \equiv \epsilon_{S} .
$$

In this notation, all $n_{\alpha}$ stand for VEVs which are singlets of the SM gauge group, all $k_{\alpha}$ are associated with SM doublets, and $\epsilon_{S}$ is the VEV of the SM triplet. Hence, in principle one expects that $\epsilon_{S} \ll k_{\alpha} \ll n_{\alpha}$.

The following Yukawa interactions are allowed by the gauge symmetry:

$$
\begin{aligned}
& \mathscr{L}_{\text {Yukawa }}=\mathscr{L}_{\text {leptons }}+\mathscr{L}_{\text {quarks }}, \\
& \mathscr{L}_{\text {leptons }}=\sum_{i=1}^{2} y_{\alpha \beta}^{\ell(i)} L_{\alpha} \ell_{\beta}^{c} \phi_{i}^{*}+y_{\alpha}^{\ell \prime} L_{e} \ell_{\alpha}^{c} S^{*}+y^{\prime \prime} L_{e} L_{e} S+\text { h.c. }, \\
& \mathscr{L}_{\text {quarks }}=\sum_{i=1}^{2} y_{\alpha \beta}^{u(i)} Q_{\alpha} u_{\beta}^{c} \phi_{i}+y_{\alpha \beta}^{d} Q_{\alpha} d_{\beta}^{c} \phi_{3}+\text { h.c. }
\end{aligned}
$$


Here, repeated Greek indices $\alpha, \beta$, representing flavors of the fermion fields, are assumed to be summed over. Fermion masses are generated from these interactions once the scalar fields acquire VEVs,

$$
\mathscr{L}_{\text {fermion mass }}=m_{\alpha \beta}^{\ell} \Psi_{\alpha}^{\ell} \Psi_{\beta}^{\ell^{c}}+m_{\alpha \beta}^{\nu} \Psi_{\alpha}^{\nu} \Psi_{\beta}^{\nu}+m_{\alpha \beta}^{u} \Psi_{\alpha}^{u} \Psi_{\beta}^{u^{c}}+m_{\alpha \beta}^{d} \Psi_{\alpha}^{d} \Psi_{\beta}^{d^{c}}+\text { h.c. . }
$$

The quark sector of the model is rather simple. Quark masses can be accommodated at tree level with a suitable choice of VEVs and Yukawa couplings $y^{u(1)}, y^{u(2)}, y^{d}$ since

$$
\begin{aligned}
& m^{u}=\left(\begin{array}{c}
y_{\alpha \beta}^{u(1)} k_{1}+y_{\alpha \beta}^{u(2)} k_{2} \\
y_{\alpha \beta}^{u(1)} n_{1}+y_{\alpha \beta}^{u(2)} n_{2}
\end{array}\right), \\
& m^{d}=y_{\alpha \beta}^{d} k_{3},
\end{aligned}
$$

in the basis $\Psi^{u}=\left(u_{\alpha}, U_{\alpha}\right)^{T}, \Psi^{u^{c}}=\left(u_{\beta}^{c}\right), \Psi^{d}=\left(d_{\alpha}\right), \Psi^{d^{c}}=\left(d_{\beta}^{c}\right)$. From equation (3.5) it is straightforward to understand why the model needs two copies of $\phi_{1,2}$ : in the case of only one $\phi$, the mass matrix $m^{u}$ has rank 3 , thus generating three massless up-quarks. On the other hand, with two copies of $\phi$ and requiring $k_{1} / n_{1} \neq k_{2} / n_{2}$, the model is able to fit the data, but there are no predictions in the quark sector.

Let us now consider the charged lepton mass matrix in the basis $\Psi^{\ell}=$ $\left(\ell_{\alpha}, E_{\alpha}, E_{e}, \ell_{e}, \Sigma^{-}\right)^{T}$ and $\Psi^{\ell^{c}}=\left(\ell_{\beta}^{c}, \Sigma^{+}\right)^{T}($ where $\alpha=\mu, \tau ; \beta=1, \cdots, 6)$ :

$$
m^{\ell}=\left(\begin{array}{cc}
y_{\alpha \beta}^{\ell(1)} k_{1}+y_{\alpha \beta}^{\ell(2)} k_{2} & 0 \\
y_{\alpha \beta}^{\ell(1)} n_{1}+y_{\alpha \beta}^{\ell(2)} n_{2} & 0 \\
n_{S} y_{\beta}^{\prime} & -\epsilon_{S} y^{\prime \prime} \\
k_{S} y_{\beta}^{\prime} & k_{S} y^{\prime \prime} \\
\epsilon_{S} y_{\beta}^{\prime} & -n_{S} y^{\prime \prime}
\end{array}\right),
$$

A striking feature of this matrix is that there is a combination of the last three rows which adds up to 0 , hence there is a massless combination of the $\Psi_{\alpha}^{\ell}$ and of the $\Psi_{\alpha}^{\ell^{c}}$ as well. The latter turns out to be a combination of the $\operatorname{six} \ell_{\beta}^{c}$ so it is a pure $(\mathbf{1}, \widehat{\mathbf{1}}, 1)$ state under the Standard Model group. As for the negatively charged massless state, it is easy to check that it corresponds to $\propto k_{S} \Sigma^{-}+\left(n_{S}-\epsilon_{S}\right) \ell_{e}-k_{S} E_{e}$. A natural possibility, although perhaps not the only one, is to associate this combination to the left-handed electron, the lightest of the charged leptons - hence the subscript $e$ in $L_{e}$. The admixture of $\Sigma^{-}$(part of an $\mathrm{SU}(2)_{L}$ triplet) and of $E_{e}$ (an $\mathrm{SU}(2)_{L}$ singlet) is suppressed by the ratio $k_{S} / n_{S}$. This ratio has to be small, due to the smallness of neutrino masses, see below. Note also that in the limit $y^{\prime \prime} \rightarrow 0$ there appears a second massless eigenstate. This limit is of course unphysical, since it corresponds to a fourth light charged lepton.

The electron mass can be generated by radiative corrections to the matrix $m^{\ell}$ - specifically to the block dependent on $y^{\prime}$. For example, the effective operator $\mathcal{O}_{\alpha}^{(i j)} L_{e} \ell_{\alpha}^{c} \phi_{i}^{*} \phi_{j} \phi_{3}$ $(i, j=1,2)$, see figure 1 , generates a contribution, roughly of the order of:

Figure 1 diagram $\propto \sum_{j=1}^{2} \frac{\lambda_{i k} k_{3}}{16 \pi^{2} \Lambda^{2}} y_{\gamma}^{\prime} y_{\delta \gamma}^{\ell(j) *} y_{\delta \alpha}^{\ell(k)} \ell_{\alpha}^{c}\left(-k_{j} n_{i} E_{e}+\frac{n_{i} n_{j}-k_{i} k_{j}}{\sqrt{2}} \ell_{e}+k_{i} n_{j} \Sigma^{-}\right)$. 


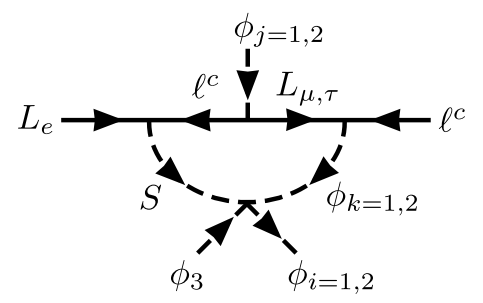

Figure 1. One-loop diagram responsible for the generation of a small electron mass.

Here, $\Lambda$ is of the order of the 331 breaking scale and $\lambda_{i j}$ is the coupling constant of the quartic scalar interaction $S \phi_{3} \phi_{i}^{*} \phi_{j}(i, j=1,2)$. Inserting very roughly $n_{i} \sim \Lambda \sim 10^{3} \mathrm{GeV}$, $k_{3} \sim 100 \mathrm{GeV}$ and all couplings $y \sim y^{\prime} \sim \lambda_{i j} \sim 0.2$ results in a mass correction of the $\mathrm{MeV}$ order.

We now turn to a discussion of neutrino masses. The fermion content of the model contains four colorless neutral fields. With the ordering $\Psi^{\nu}=\left(\nu_{\mu}, \nu_{\tau}, \nu_{e}, \Sigma^{0}\right)^{T}$ the tree-level mass matrix reads

$$
m^{\nu}=y^{\prime \prime}\left(\begin{array}{cccc}
0 & 0 & 0 & 0 \\
0 & 0 & 0 & 0 \\
0 & 0 & \epsilon_{S} & -\frac{k_{S}}{\sqrt{2}} \\
0 & 0 & -\frac{k_{S}}{\sqrt{2}} & n_{S}
\end{array}\right),
$$

i.e. at tree-level there are two massless neutrinos, $\nu_{\mu}$ and $\nu_{\tau}$. In the seesaw approximation, the two non-zero eigenstates have masses $m_{\Sigma} \simeq y^{\prime \prime} n_{S}$ and $m_{\nu} \simeq y^{\prime \prime}\left[\epsilon_{S}-k_{S}^{2} /\left(2 n_{S}\right)\right]$. The latter is a mixture of a type-II and type-III seesaw mechanism contributions. Unless one considers the extremely fine-tuned situation where $\epsilon_{S} \equiv k_{S}^{2} /\left(2 n_{S}\right)$, this implies that $k_{S}$ has to much smaller than all other $k_{i}$, i.e. for $n_{S} \sim \mathrm{TeV}$ one needs $k_{S}$ to be below $10^{-3} \mathrm{GeV}$.

This tree-level picture is clearly insufficient, since oscillation data requires (a) that at least two neutrinos should be massive, and (b) that $\nu_{e}$ mixes with the two other light neutrinos. We thus have also to consider loops contributing to the neutrino mass matrix. We are especially interested in effective operators of the type $\mathcal{O}_{\alpha}^{\prime(i)} / \Lambda L_{\alpha} L_{e} \phi_{i} S(\alpha=\mu, \tau$; $i=1,2)$, since these will generate contributions mixing $\nu_{e}$ with $\nu_{\mu}$ and $\nu_{\tau}$. Indeed, as shown in figure 2, such type of operator is already present in the model, using only the minimal set of scalars given in table 2 . A rough estimate of this diagram results in

Figure 2 diagram
$\propto \sum_{j=1}^{2} \frac{f_{i j}}{16 \pi^{2} \Lambda^{2}} y_{\alpha \beta}^{\ell(j)} y_{\beta}^{\ell \prime *} y^{\prime \prime} \nu_{\alpha}\left[\left(-\frac{k_{i} k_{S}}{\sqrt{2}}+n_{i} \epsilon_{S}\right) \nu_{e}+\left(-\frac{n_{i} k_{S}}{\sqrt{2}}+k_{i} n_{S}\right) \Sigma^{0}\right]$
$\equiv \omega_{e \mu} \nu_{e} \nu_{\mu}+\omega_{e \tau} \nu_{e} \nu_{\tau}+\omega_{\Sigma \mu} \nu_{\Sigma} \nu_{\mu}+\omega_{\Sigma \tau} \nu_{\Sigma} \nu_{\tau}$

where $f_{i j}=f_{j i}$ represents the coupling constant of the trilinear scalar interaction $\phi_{i} \phi_{j} S$ $(i, j=1,2)$. Note that equation (3.10) does not include the corresponding loop functions and thus serves only as a rough order-of-magnitude estimate.

There is sufficient freedom in the Yukawa couplings and the VEVs to consider the four $\omega_{\alpha \beta}$ coefficients to be essentially free parameters. Inserting the $\omega_{\alpha \beta}$ coefficients in 


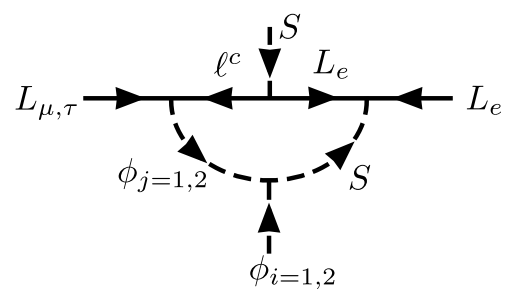

Figure 2. Loop diagram responsible for the generation of two of the light neutrino masses as well as the mixing of $\nu_{e}$ with the other two light neutrinos.

the matrix $m^{\nu}$ shown in equation (3.9), and integrating out the heavy $\Sigma^{0}$ state with the standard seesaw formula, we obtain

$$
m_{\text {light }}^{\nu} \approx\left(\begin{array}{ccc}
\epsilon_{S}-\frac{k_{S}^{2}}{2 n_{S}} & \omega_{e \mu}+\omega_{\Sigma \mu} \frac{k_{S}}{\sqrt{2} n_{S}} \omega_{e \tau}+\omega_{\Sigma \tau} \frac{k_{S}}{\sqrt{2} n_{S}} \\
\omega_{e \mu}+\omega_{\Sigma \mu} \frac{k_{S}}{\sqrt{2} n_{S}} & -\frac{\omega_{\Sigma \mu}^{2}}{n_{S}} & -\frac{\omega_{\Sigma \mu} \omega_{\Sigma \tau}}{n_{S}} \\
\omega_{e \tau}+\omega_{\Sigma \tau} \frac{k_{S}}{\sqrt{2} n_{S}} & -\frac{\omega_{\Sigma \mu} \omega_{\Sigma \tau}}{n_{S}} & -\frac{\omega_{\Sigma \tau}^{2}}{n_{S}}
\end{array}\right)
$$

in the $\left(\nu_{e}, \nu_{\mu}, \nu_{\tau}\right)$ basis, to a good approximation (for simplicity, we ignore here the charged lepton rotation matrix, which at tree level only affects the $\mu \tau$ sector). Notice that the structure in the $\mu \tau$ block of this matrix has determinant zero. Neutrino oscillation data [14] can be fitted to a matrix with such a property, but the fit strongly prefers inverse hierarchy with a small but slightly non-zero value for $m_{\nu_{3}}$. One can also easily check that for $y_{\alpha \beta}^{\ell(j)} \sim$ $y_{\beta}^{\ell \prime} \sim \mathcal{O}\left(10^{-1}\right), y^{\prime \prime} \sim \mathcal{O}(1)$, the scales $k_{i} \sim 200 \mathrm{GeV}, n_{S} \sim \Lambda \sim \mathcal{O}(\mathrm{TeV})$ and $k_{S} \sim 10^{-4} \mathrm{GeV}$, one requires roughly $f_{i j} \sim 10 \mathrm{GeV}$ in order to get entries in the neutrino mass matrix of order few 10's of $\mathrm{meV}$, the magnitude required by the experimental data for inverse hierarchical neutrinos.

Thus, the minimal model, as defined in table 2, predicts an inverse hierarchy for neutrinos. However, it should be kept in mind that with more scalars added to the model, other fits to neutrino data will become possible as well.

Finally, we turn our attention to flavor changing neutral current effects in the quark and charged lepton sectors. In addition to the Standard Model photon and $Z$ boson, 331 models contain a $Z^{\prime}$ boson with fermion interactions which are essentially diagonal in the flavor basis. In standard 331 models, since all three lepton families are in the same group representation, there are no $Z^{\prime}$ lepton flavor changing interactions. On the other hand, left-handed quarks are placed in one triplet plus two anti-triplet representations of $\mathrm{SU}(3)_{L}$. For the $Z^{\prime}$ quark-antiquark interactions there appears then a matrix $\eta=\operatorname{diag}(1,-1,-1)$ in the flavor basis which, once the quarks are rotated to the mass eigenstate basis, produces a vertex proportional to the combination $V^{\dagger} \eta V$, where $V$ is the rotation matrix for lefthanded quarks. Thus, the GIM mechanism does not work in $Z^{\prime}$ interactions with quarks in standard 331 models. This results in stringent lower limits on the $Z^{\prime}$ mass [15].

In our model, the situation is reversed. Now all left-handed quark families are in anti-triplet representations, thus the only limits on $Z^{\prime}$ from FCNC observables come from terms mixing ordinary with exotic quarks. Note that this mixing can be made arbitrarily 


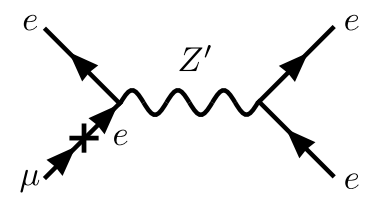

Figure 3. The $\mu \rightarrow e \bar{e} e$ decay mediated by the $Z^{\prime}$ gauge boson in the flipped 331 model.

small [16]. For this reason, anomalies in rare kaon an $B$ decays cannot be explained by $Z^{\prime}$ interactions in our setup. However, since leptons are in different multiplets in our model, now potentially dangerous $Z^{\prime}$ interactions will appear in the lepton sector (see figure 3 ). In the flavor basis, the relevant Lagrangian term is

$$
\mathscr{L}_{\ell Z^{\prime}}=i\left(\sqrt{3 g_{L}^{2}-g_{Y}^{2}} y_{\alpha}-\frac{3 g_{L}^{2}}{\sqrt{3 g_{L}^{2}-g_{Y}^{2}}} x_{\alpha}\right) \bar{\ell}_{\alpha} \gamma^{\mu} \ell_{\alpha} Z_{\mu}^{\prime}
$$

with $y_{e, \mu, \tau}=-1 / 2, x_{e}=-1 / 3$ and $x_{\mu, \tau}=-2 / 3$. After rotation to the mass basis, with a matrix $V_{l_{\alpha} l_{\beta}}(\alpha, \beta=e, \mu, \tau)$, we find:

$$
\begin{aligned}
\mathscr{L}_{\ell Z^{\prime}} & =i\left(C_{e e} \bar{e}_{L} \gamma^{\mu} e_{L}+C_{e \mu} \bar{e}_{L} \gamma^{\mu} \mu_{L}\right) Z_{\mu}^{\prime} \\
\text { with } C_{e e} & \approx \frac{g_{Y}^{2}-g_{L}^{2}}{2 \sqrt{3 g_{L}^{2}-g_{Y}^{2}}} \quad \text { and } C_{e \mu}=-\frac{g_{L}^{2}}{\sqrt{3 g_{L}^{2}-g_{Y}^{2}}} V_{\ell_{e} \ell_{\mu}},
\end{aligned}
$$

so $[17]$

$$
\operatorname{Br}(\mu \rightarrow e \bar{e} e)=\left|\frac{C_{e e} C_{e \mu}}{G_{F} m_{Z^{\prime}}^{2}}\right|^{2} \approx 8\left(\frac{1-2 \sin ^{2} \theta_{w}}{3-4 \sin ^{2} \theta_{w}}\right)^{2}\left|V_{\ell_{e} \ell_{\mu}}\right|^{2}\left(\frac{m_{W}}{m_{Z^{\prime}}}\right)^{4}
$$

However, recall that $\ell_{e}$ and $\ell_{\mu}$ do not mix at tree level. After taking into account radiative corrections, one expects that not only a non-zero electron mass will appear, but also a non-zero mixing element $V_{\ell_{e} \ell_{\mu}}$, of the order of $m_{e} / m_{\mu}$, will be generated. Thus, we obtain the simple estimate

$$
\operatorname{Br}(\mu \rightarrow e \bar{e} e) \lesssim 10^{-12}\left(\frac{5 \mathrm{TeV}}{m_{Z^{\prime}}}\right)^{4}\left|\frac{V_{\ell_{e} \ell_{\mu}}}{\left(m_{e} / m_{\mu}\right)}\right|^{2}
$$

Given that this branching ratio is known to be smaller than approximately $10^{-12}$ [18], it provides a lower bound on the $Z^{\prime}$ mass comparable to the LHC one $\left(m_{Z^{\prime}} \gtrsim 3 \mathrm{TeV}\right)[19,20]$. Note that equation (3.15) depends quadratically on $V_{\ell_{e} \ell_{\mu}}$.

In models such as this one, the $Z$ boson mass eigenstate has a slightly different composition than in the Standard Model, which is a consequence of the so-called $Z-Z^{\prime}$ mixing. In practice, this means that there is an analogue to the diagram in figure 3 where the $Z^{\prime}$ is replaced by a $Z$ boson. However, the $Z_{\nu} \bar{\mu}_{L} \gamma^{\nu} e_{L}$ interaction is suppressed by a factor 
$\rho m_{Z}^{2} / m_{Z^{\prime}}^{2}$ relative to the $Z_{\nu}^{\prime} \bar{\mu}_{L} \gamma^{\nu} e_{L}$ interaction. It turns out that $|\rho|<1$, so at most we expect a $\mathcal{O}(1)$ correction to equation (3.15) due to $Z$ - $Z^{\prime}$ mixing. ${ }^{3}$

We mention in passing that there will also be some constraints from other decays such as $\ell_{i} \rightarrow \ell_{j} \gamma$, which are induced by loops with the heavy gauge bosons or the heavy fermions. However, we expect the decay $\mu \rightarrow e \bar{e} e$ to give the most stringent bound on the 331 breaking scale.

\section{Summary}

This work describes for the first time a model based on the $\mathrm{SU}(3)_{C} \times \mathrm{SU}(3)_{L} \times \mathrm{U}(1)_{X}$ gauge group where all three quark families are placed in equal representations of the gauge group, while the lepton families are not. The required number of fermions representations is the same as in a standard 331 model. There are no gauge anomalies, and the observed fermion masses and mixings can be accommodated with a minimal scalar sector composed of three triplets and a sextet. Unlike in standard 331 models where the parameter $\beta$ controlling the relation $Y=\beta T_{8}+X$ can take more than one value, in our flipped 331 construction $\beta$ must be $1 / \sqrt{3}$.

With the minimal setup considered in this paper, one charged lepton (the electron) and two neutrinos are massless at tree level. Radiative effects can generate the missing masses and mixing angles, favoring an inverse neutrino mass hierarchy, with $\nu_{3}$ almost massless. If the scalar sector of the model is extended, other constructions might be possible.

As in all standard 331 models, there are potentially dangerous flavor violating $Z^{\prime}$ interactions. However, unlike in other 331 models, in our flipped scenario there are no constraints from the quark sector, and $Z^{\prime}$ interactions in the model cannot explain the known hints of anomalies in $K$ and $B$ physics. Instead, amplitudes of lepton number violating processes are expected to be sizable. For example, if the $Z^{\prime}$ is observed at the LHC, we expect that the proposed Mu3e experiment [21] would very likely observe the decay $\mu \rightarrow e \bar{e} e$.

\section{Acknowledgments}

This work was supported by the Spanish grants FPA2014-58183-P, Multidark CSD200900064 and SEV-2014-0398 (from the Ministerio de Economía y Competitividad), as well as PROMETEOII/2014/084 (from the Generalitat Valenciana).

Open Access. This article is distributed under the terms of the Creative Commons Attribution License (CC-BY 4.0), which permits any use, distribution and reproduction in any medium, provided the original author(s) and source are credited.

\footnotetext{
${ }^{3}$ Given that the interaction of the $Z$ to electrons is somewhat stronger than the one of $Z^{\prime}$, even though $\rho$ can never be -1 , it still seems possible, even if improbable, to have a complete suppression of the $\mu \rightarrow e \bar{e} e$ process. Note that a rigorous analysis of such a delicate cancellation would require also taking into account the decay of the muon to right-handed electrons which, for simplicity, was not included in equation (3.14).
} 


\section{References}

[1] J. Schechter and Y. Ueda, Unified weak-electromagnetic gauge schemes based on the three-dimensional unitary group, Phys. Rev. D 8 (1973) 484 [INSPIRE].

[2] V. Gupta and H.S. Mani, Unified weak and electromagnetic gauge theory based on $\mathrm{SU}(3) \times \mathrm{U}(1)$, Phys. Rev. D 10 (1974) 1310 [inSPIRE].

[3] C.H. Albright, C. Jarlskog and M.O. Tjia, Implications of Gauge Theories for Heavy Leptons, Nucl. Phys. B 86 (1975) 535 [inSPIRE].

[4] H. Georgi and A. Pais, Generalization of Gim: Horizontal and Vertical Flavor Mixing, Phys. Rev. D 19 (1979) 2746 [INSPIRE].

[5] M. Singer, J.W.F. Valle and J. Schechter, Canonical Neutral Current Predictions From the Weak Electromagnetic Gauge Group SU(3) $\times \mathrm{U}(1)$, Phys. Rev. D 22 (1980) 738 [INSPIRE].

[6] F. Pisano and V. Pleitez, An SU(3) $\times \mathrm{U}(1)$ model for electroweak interactions, Phys. Rev. D 46 (1992) 410 [hep-ph/9206242] [INSPIRE].

[7] P.H. Frampton, Chiral dilepton model and the flavor question, Phys. Rev. Lett. 69 (1992) 2889 [INSPIRE].

[8] R.A. Diaz, R. Martinez and F. Ochoa, $\mathrm{SU}(3)_{c} \times \mathrm{SU}(3)_{L} \times \mathrm{U}(1)_{X}$ models for beta arbitrary and families with mirror fermions, Phys. Rev. D 72 (2005) 035018 [hep-ph/0411263] [INSPIRE].

[9] A.J. Buras, F. De Fazio and J. Girrbach-Noe, $Z$-Z' mixing and $Z$-mediated FCNCs in $\mathrm{SU}(3)_{C} \times \mathrm{SU}(3)_{L} \times \mathrm{U}(1)_{X}$ models, JHEP 08 (2014) 039 [arXiv:1405.3850] [InSPIRE].

[10] R. Foot, O.F. Hernandez, F. Pisano and V. Pleitez, Lepton masses in an $\mathrm{SU}(3)_{L} \times \mathrm{U}(1)_{N}$ gauge model, Phys. Rev. D 47 (1993) 4158 [hep-ph/9207264] [INSPIRE].

[11] R.M. Fonseca and M. Hirsch, Lepton number violation in 331 models, arXiv:1607.06328 [INSPIRE].

[12] L.A. Sanchez, W.A. Ponce and R. Martinez, $\mathrm{SU}(3)_{c} \times \mathrm{SU}(3)_{L} \times \mathrm{U}(1)_{X}$ as an $E_{6}$ subgroup, Phys. Rev. D 64 (2001) 075013 [hep-ph/0103244] [INSPIRE].

[13] R.M. Fonseca, On the chirality of the SM and the fermion content of GUTs, Nucl. Phys. B 897 (2015) 757 [arXiv: 1504.03695] [INSPIRE].

[14] D.V. Forero, M. Tortola and J.W.F. Valle, Neutrino oscillations refitted, Phys. Rev. D 90 (2014) 093006 [arXiv:1405.7540] [INSPIRE].

[15] A.J. Buras and F. De Fazio, $\varepsilon^{\prime} / \varepsilon$ in 331 Models, JHEP 03 (2016) 010 [arXiv:1512.02869] [INSPIRE].

[16] S.M. Boucenna, J.W.F. Valle and A. Vicente, Predicting charged lepton flavor violation from 3-3-1 gauge symmetry, Phys. Rev. D 92 (2015) 053001 [arXiv:1502.07546] [INSPIRE].

[17] Y. Kuno and Y. Okada, Muon decay and physics beyond the standard model, Rev. Mod. Phys. 73 (2001) 151 [hep-ph/9909265] [INSPIRE].

[18] SINDRUM collaboration, U. Bellgardt et al., Search for the Decay $\mu+\rightarrow e^{+} e^{+} e^{-}$, Nucl. Phys. B 299 (1988) 1 [inSPIRE].

[19] ATLAS collaboration, Search for new phenomena in the dilepton final state using proton-proton collisions at $\sqrt{s}=13$ TeV with the ATLAS detector, ATLAS-CONF-2015-070 (2015).

[20] CMS collaboration, Search for a Narrow Resonance Produced in 13 TeV pp Collisions Decaying to Electron Pair or Muon Pair Final States, CMS-PAS-EXO-15-005 (2015).

[21] A. Blondel et al., Research Proposal for an Experiment to Search for the Decay $\mu \rightarrow$ eee, arXiv: 1301.6113 [INSPIRE]. 